\title{
Concomitant Tricuspid Valve Repair during Minimally Invasive Mitral Valve Repair
}

\author{
Bettina Pfannmueller ${ }^{1} \quad$ Martin Misfeld $^{1} \quad$ Piroze Davierwala $^{1} \quad$ Stefan Weiss $^{2} \quad$ Michael Andrew Borger $^{1}$ \\ ${ }^{1}$ Department of Heart Surgery, Leipzig Heart Centre, Leipzig \\ University Hospital, Leipzig, Germany \\ ${ }^{2}$ Leipzig Heart Institute $\mathrm{GmbH}$, Leipzig, Germany \\ Thorac Cardiovasc Surg 2020;68:486-491. \\ Address for correspondence Bettina Pfannmueller, MD, PhD, \\ Department of Heart Surgery, Leipzig Heart Centre, Leipzig University \\ Hospital, Strümpellstraße 39, 04289 Leipzig, Germany \\ (e-mail: bettina.pfannmueller@medizin.uni-leipzig.de).
}

\begin{abstract}
Keyword

- mitral valve surgery

- tricuspid valve

- minimally invasive surgery

Background Concomitant use of tricuspid valve (TV) surgery and minimally invasive mitral valve (MV) repair is debatable due to a prolonged time of surgery with presumably elevated operative risk. Herein, we examined cardiopulmonary bypass times and 30-day mortality in patients who underwent MV repair with and without concomitant TV surgery.

Methods We retrospectively evaluated 3,962 patients with MV regurgitation who underwent minimally invasive MV repair without ( $n=3,463$; MVr group) and with ( $n=499 ; \mathrm{MVr}+\mathrm{TV} r$ group) concomitant TV surgery between 1999 and 2014. Preoperative parameters between the groups were significantly different; therefore, propensity score matching was performed.

Results Mean cardiopulmonary bypass time for all patients was $125.5 \pm 55.8$ minutes in $\mathrm{MVr}$ and $162.0 \pm 58.0$ minutes in $\mathrm{MVr}+\mathrm{TVr}(p<0.001)$. Overall 30-day mortality was significantly different between these groups ( 4.8 vs. $2.1 \%$; $p<0.001)$; however, after adjustment, there was no significant difference ( 3.3 vs. $1.2 \% ; p=0.07$ ). Backward logistic regression revealed that cardiopulmonary bypass time was not a significant predictor for early mortality within the $\mathrm{MVr}+\mathrm{TV} r$ cohort.

Conclusion Concomitant TV repair using prosthetic rings through a minimally invasive approach is safe and does not lead to elevated early mortality in our patient cohort. Therefore, prolonged cardiopulmonary bypass time should not be the sole reason to rule out MV repair with concomitant TV repair and to prefer the use of suture techniques, which saves only a few minutes compared with prosthetic ring implantation.
\end{abstract}

\section{Introduction}

The European guidelines for the management of valvular heart disease recommend the use of mitral valve (MV) surgery with concomitant tricuspid valve (TV) surgery in cases of dilated TV annulus and/or TV regurgitation $\geq$ grade II. ${ }^{1}$ Nevertheless, there are different surgical concepts. There are concerns regarding operative risk due to prolonged cardiopulmonary bypass time during concomitant TV sur-

received

March 16, 2019

accepted after revision

September 9, 2019

published online

December 31, 2019

gery, especially that using a minimally invasive approach. Moreover, there are concerns regarding the repair technique itself as to whether it would be better to avoid implantation of a prosthetic ring with respect to performing a suture annuloplasty to save time in case of concomitant TV surgery. The latter is pointed out in a study on the prosthetic ring annuloplasty of TV: "However, this procedure leads to some prolongation of the operation and cardiopulmonary bypass time. Therefore, intervening on moderate functional TR in

(c) 2020 Georg Thieme Verlag KG Stuttgart · New York
Dol https://doi.org/ 10.1055/s-0039-1700506. ISSN 0171-6425. 
the context of another cardiac procedure may become a decision making dilemma. For such cases, a modified suture annuloplasty technique was developed..."2

Therefore, there are several important questions, which we aimed to answer in this study, such as how much time does the prosthetic annuloplasty ring of TV take during minimally invasive procedure, what is the average difference in cardiopulmonary bypass time between patients undergoing isolated MV repair and those undergoing additional TV repair, and does prolonged cardiopulmonary bypass times augment operative risk during minimally invasive MV repair with concomitant TV repair?

\section{Patients and Methods}

We retrospectively analyzed 3,962 patients who underwent minimally invasive MV repair (MVr) and maze procedure without ( $n=3,463$; MVr group) and with concomitant TV surgery using a prosthetic annuloplasty ring ( $n=499$; MVr + TVr group). Patients with MV and/or TV replacements, those with TV repair without using prosthetic rings, those with active endocarditis, and those with intraoperative conversion to sternotomy were excluded from the study.

\section{Data Collection}

Relevant preoperative, intraoperative, and postoperative outcome data of all patients were prospectively entered into the institutional database and retrospectively analyzed. Completely anonymized data were retrieved from the hospital data collecting system.

\section{Indications for Tricuspid Valve Repair}

Indications for TV repair were as follows: TV annulus dilated $>40 \mathrm{~mm}$ or/and the presence of TR $\geq$ grade II. TR was classified by our institutional echocardiography laboratory on the basis of the vena contracta in a four-chamber view according to the European guidelines on the management of valvular heart disease. ${ }^{3}$

\section{Surgical Procedure}

We used a minimally invasive standard approach through a right anterolateral minithoracotomy, which has been previously described in detail by our group. ${ }^{4-7}$ For minimally invasive MV repair, we routinely cannulated the right femoral artery and vein in the groin for cardiopulmonary bypass. For TV repair, we cannulated the internal jugular vein for additional venous drainage of the upper body. TV repair was performed with the beating heart or by applying the crossclamp at the discretion of the surgeon.

\section{Statistical Evaluation}

Categorical variables were reported as count (percentage). Pearson's $\chi^{2}$ test was used for group comparisons.

Continuous study variables were tested for normal distribution and were reported as median and interquartile range in case of violation or as mean and standard deviation otherwise.

For metric variables, the statistical test used was assessed by variance of heterogeneity, and normality and was chosen between unpaired $t$-test, unpaired Welch's $t$-test, and Wilcoxon's signed-rank test.

Bootstrapped backward stepwise logistic regression was performed for bypass time and preoperative characteristics (-Table 1) for patients undergoing minimally invasive MV repair and concomitant TV repair to evaluate risk factors for this patient population.

Greedy nearest-neighbor 1:1 propensity score matching with a caliper of 0.2 was applied to variables age, body mass

Table 1 Preoperative parameters of the cohort

\begin{tabular}{|l|l|l|l|l|l|}
\hline Variable & $\begin{array}{l}\text { All patients } \\
(\boldsymbol{n}=3,962)\end{array}$ & $\begin{array}{l}\text { Isolated MV surgery } \\
(\boldsymbol{n}=3,463)(\mathrm{MVr})\end{array}$ & $\begin{array}{l}\text { MV surgery+TV repair } \\
(\boldsymbol{n}=499)(\mathrm{MVr}+\mathrm{TV})\end{array}$ & $\begin{array}{l}\boldsymbol{p} \text {-Value } \\
(\text { before } \\
\text { matching) }\end{array}$ & $\begin{array}{l}\boldsymbol{p} \text {-Value } \\
\text { (after matching) }\end{array}$ \\
\hline Age (years) & $61.6(51.3-70.5)$ & $59.76(50.1-69.1)$ & $70.87(64.0-75.6)$ & $<0.001$ & n.s. \\
\hline Gender, male & $2,473(62.4 \%)$ & $2,232(64.5 \%)$ & $241(48.3 \%)$ & $<0.001$ & n.s \\
\hline LVEF (\%) & $59.8( \pm 12.5)$ & $60.4( \pm 12.3)$ & $55.2( \pm 13.0)$ & $<0.001$ & n.s \\
\hline Atrial fibrillation & $1,250(31.6 \%)$ & $925(26.7 \%)$ & $325(65.1 \%)$ & $<0.001$ & n.s \\
\hline $\begin{array}{l}\text { Arterial } \\
\text { hypertension }\end{array}$ & $2,559(64.6 \%)$ & $2,140(61.8 \%)$ & $419(84.0 \%)$ & $<0.001$ & n.s \\
\hline $\begin{array}{l}\text { Pulmonary } \\
\text { hypertension }\end{array}$ & $679(17.1 \%)$ & $493(14.2 \%)$ & $186(37.2 \%)$ & $<0.001$ & n.s \\
\hline COPD & $147(3.7 \%)$ & $111(3.2 \%)$ & $36(7.2 \%)$ & $<0.001$ & n.s \\
\hline Diabetes mellitus & $407(10.3 \%)$ & $316(9.1 \%)$ & $91(18.2 \%)$ & $<0.001$ & n.s \\
\hline Dialysis & $33(0.8 \%)$ & $19(0.6 \%)$ & $14(2.8 \%)$ & $<0.001$ & n.s \\
\hline BMl (kg/m $\left.{ }^{2}\right)$ & $25.7( \pm 4.0)$ & $25.6( \pm 3.9)$ & $26.0( \pm 4.3)$ & 0.0204 & n.s \\
\hline $\begin{array}{l}\text { Previous } \\
\text { cardiac surgery }\end{array}$ & $156(3.9 \%)$ & $135(3.9 \%)$ & $21(4.2 \%)$ & n.s. & n.s \\
\hline
\end{tabular}

Abbreviations: BMI, body mass index; COPD, chronic obstructive pulmonary disease; LVEF, left ventricular ejection fraction; MV, mitral valve; MVr, mitral valve repair; n.s., not significant; TV, tricuspid valve; TVr, tricuspid valve repair. 
Table 2 Preoperative MV pathology

\begin{tabular}{|c|c|c|c|c|}
\hline & $\begin{array}{l}\text { All patients } \\
(n=3,962)\end{array}$ & $\begin{array}{l}\text { Isolated MV surgery } \\
(n=3,463)(\mathrm{MVr})\end{array}$ & $\begin{array}{l}\text { MV surgery }+ \text { TV repair } \\
(n=499)(\mathrm{MVr}+\mathrm{TVr})\end{array}$ & $p$-Value \\
\hline MV stenosis & $35(0.9 \%)$ & $32(0.9 \%)$ & $3(0.6 \%)$ & n.s. \\
\hline Annulus dilatation & $3,384(85.4 \%)$ & $2,944(85.0 \%)$ & $440(88.2 \%)$ & n.s. \\
\hline Annulus calcification & $108(2.7 \%)$ & $90(2.6 \%)$ & $18(3.6 \%)$ & n.s. \\
\hline Prolapse PML & $2,600(65.6 \%)$ & $2,423(70.0 \%)$ & 177 (35.5\%) & $<0.001$ \\
\hline Prolapse AML & $1,066(26.9 \%)$ & $957(27.6 \%)$ & $109(21.8 \%)$ & $<0.01$ \\
\hline Leaflet restriction & $228(5.8 \%)$ & $180(5.2 \%)$ & $48(9.6 \%)$ & $<0.001$ \\
\hline Pacemaker & $197(5.0 \%)$ & $136(4.0 \%)$ & $62(12.2 \%)$ & $<0.001$ \\
\hline
\end{tabular}

Abbreviations: AML, anterior mitral leaflet; MV, mitral valve; MVr, mitral valve repair; n.s., not significant; PML, posterior mitral leaflet; TV, tricuspid valve; TVr, tricuspid valve repair.

index, gender, diabetes, prior cardiac surgery, diabetes, chronic obstructive pulmonary disease, creatinine, arterial and pulmonary hypertension, priority status, peripheral vascular disease, atrial fibrillation, and pacemaker, stratifying 467 patients by treatment. Differences in early mortality and perioperative outcomes were tested thereafter.

Statistical analyses were performed using the SPSS statistical package, version 20.0 (SPSS Inc., Chicago, Illinois, United States) and validated in $\mathrm{R}$, version 3.5.1. A p-value of $<0.05$ was considered statistically significant.

\section{Results}

Mean patients age was 62 (51-71) years, and 62\% of the patients were male $(n=2,473)$. Average log EuroSCORE was $2.7(1.5-5.5) \%$, and the average left ventricle ejection fraction (LVEF) was $60 \pm 13 \%$. In total, $10 \%$ of the patients suffered from diabetes mellitus $(n=407)$ and $32 \%$ from atrial fibrillation $(n=1,250)$. In $5 \%$ of the patients, a previous pacemaker was implanted ( $n=197)$, and in $4 \%$ of patients, previous cardiac surgery has been performed $(n=156)$.

Preoperative parameters were significantly different between groups (-Table $\mathbf{1}$ ). $\mathrm{MVr}+\mathrm{TVr}$ patients were on average 10 years older, had lower LVEF, and were mostly females, compared with the MVr-group. In the $\mathrm{MVr}+\mathrm{TVr}$ group, the number of patients with diabetes mellitus and atrial fibrillation was twofold higher and the number of patients with a pacemaker was threefold higher than those in the $\mathrm{MVr}$ group. The number of patients with a history of cardiac surgery was comparable between both groups.
In the $\mathrm{MVr}+\mathrm{TVr}$ group, more patients presented with a functional pathology of MV (-Table 2). Mitral leaflet repair was more common in isolated $\mathrm{MVr}$, whereas cryoablation due to atrial fibrillation was more common in the $\mathrm{MVr}+\mathrm{TVr}$ group (MVr: 22.9\%; MVr + TVr: 54.7\%; $p<0.001$ ); MV annuloplasty was usually performed with rigid rings ( $n=3,395$; 85.7\%) and TV annuloplasty with flexible bands ( $n=385$; $77.2 \%)$.

Comparison of cross-clamp time, bypass time, and surgery time revealed significant differences between both groups (prolonged in $\mathrm{MVr}+\mathrm{TVr}$ patients; $p<0.001$ ), with average differences of 18,37 , and 41 minutes, respectively (-Table 3 ). Outliers in bypass time were less frequent in patients with early mortality (EM) (-Fig. 1), and there was noted a difference of 44 minutes in bypass time between patients who underwent MV repair with and without TV surgery.

Early mortality was significantly higher in the $\mathrm{MVr}+\mathrm{TVr}$ group ( 4.8 vs. $2.1 \% ; p<0.001$ ) before matching. After matching, early mortality was not significantly different between both groups (4.71 vs. 3.21\%; $p=0.2403$ ). ICU stay, ventilation time, and hospital stay was longer in the $\mathrm{MVr}+\mathrm{TVr}$ group than in the MVr group $(p<0.0001)$, and this was also seen after matching (-Tables 4 and $\mathbf{5}$ ).

Intensive care unit (ICU) stay and ventilation times were each around 5 hours prolonged in patients with additionally TV repair (after matching), which was significantly different (-Table 5).

Binomial logistic regression model after matching revealed LVEF (odds ratio $[\mathrm{OR}]=0.95$; confidence interval $[\mathrm{CI}]=0.91-$ $0.98 ; p=0.0047$ ) as a predictor for early mortality in the $\mathrm{MVr}$

Table 3 Intraoperative data

\begin{tabular}{|c|c|c|c|c|}
\hline & $\begin{array}{l}\text { All patients } \\
(n=3,962)\end{array}$ & $\begin{array}{l}\text { Isolated MV surgery } \\
(n=3,463)(M V r)\end{array}$ & $\begin{array}{l}\text { MV surgery }+ \text { TV } \\
\text { repair }(n=499) \\
(\mathrm{MVr}+\mathrm{TVr})\end{array}$ & $p$-Value \\
\hline Cross-clamp time (minutes) & $76.1 \pm 31.9$ & $74.9 \pm 32.0$ & $92.8 \pm 30.3$ & $<0.001$ \\
\hline Cardiopulmonary bypass time (minutes) & $130.3 \pm 37.8$ & $127.04 \pm 36.1$ & $164.1 \pm 34.0$ & $<0.001$ \\
\hline Time for surgery (minutes) & $178.2 \pm 48.0$ & $174.2 \pm 46.2$ & $215.5 \pm 42.3$ & $<0.001$ \\
\hline
\end{tabular}

Abbreviations: MV, mitral valve; MVr, mitral valve repair; TV, tricuspid valve; TVr, tricuspid valve repair. 


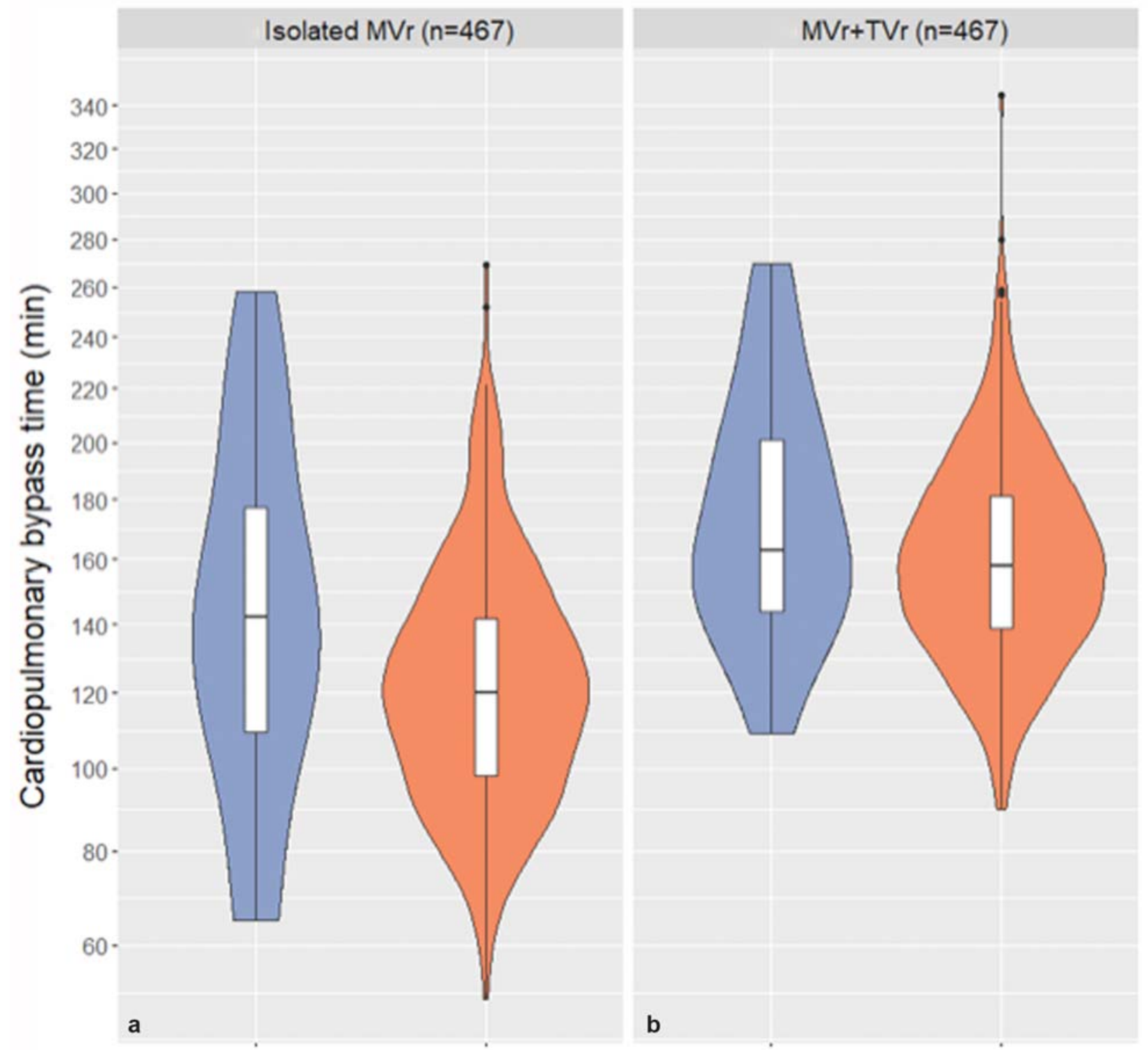

Early Mortality Surviving patients Early Mortality Surviving patients

Fig. 1 (a) A violin plot showing the distribution and outliers of cardiopulmonary bypass time for the matched cohort: patients who died within 30 days after operation versus surviving patients (isolated MVr). (b) A violin plot showing the distribution and outliers of cardiopulmonary bypass time for the matched cohort: patients who died within 30 days after operation versus surviving patients ( $M V r+T V r)$. MVr, mitral valve repair; $\mathrm{TV}$, tricuspid valve repair.

Table 4 Postoperative data after matching

\begin{tabular}{|l|l|l|l|l|}
\hline & $\begin{array}{l}\text { All patients } \\
(\boldsymbol{n}=3,962)\end{array}$ & $\begin{array}{l}\text { Isolated MV surgery } \\
(\boldsymbol{n}=3,463)(\mathrm{MVr})\end{array}$ & $\begin{array}{l}\text { MV surgery+TV repair } \\
(\boldsymbol{n}=\mathbf{4 9 9})(\mathrm{MVr}+\mathrm{TVr})\end{array}$ & $\boldsymbol{p}$-Value \\
\hline ICU time (hours) & $21.9(8.7-46.4)$ & $19.8(5.7-27.6)$ & $24.2(16.3-69.7)$ & $<0.0001$ \\
\hline Ventilation time (hours) & $13.9(8.6-27.0)$ & $11.9(7.4-20.2)$ & $16.8(9.8-52.2)$ & $<0.0001$ \\
\hline Hospital stay (days) & $12(9-17)$ & $11(9-15)$ & $13(10-19)$ & $<0.0001$ \\
\hline 30-d mortality & $37(4.0 \%)$ & $15(3.2 \%)$ & $22(4.7 \%)$ & 0.2403 \\
\hline
\end{tabular}

Abbreviations: ICU, intensive care unit; MV, mitral valve; MVr, mitral valve repair; TV, tricuspid valve; TVr, tricuspid valve repair. 
Table 5 Postoperative data before matching

\begin{tabular}{|l|l|l|l|l|}
\hline & All patients $(\boldsymbol{n}=\mathbf{3 , 9 6 2 )}$ & $\begin{array}{l}\text { Isolated MV surgery } \\
(\boldsymbol{n}=3,463)(\mathrm{MVr})\end{array}$ & $\begin{array}{l}\text { MV surgery+ TV repair } \\
(\boldsymbol{n}=\mathbf{4 9 9})(\mathrm{MVr}+\mathrm{TVr})\end{array}$ & $\boldsymbol{p}$-Value \\
\hline ICU time (hours) & $15.9(2.6-24.4)$ & $11.3(0.0-23.3)$ & $24.2(16.3-69.3)$ & $<0.0001$ \\
\hline Ventilation time (hours) & $9.6(6.0-17.8)$ & $8.7(5.6-15.9)$ & $16.5(9.7-50.5)$ & $<0.0001$ \\
\hline Hospital stay (days) & $10(8-13)$ & $9(8-12)$ & $13(10-19)$ & $<0.0001$ \\
\hline 30-d mortality & $96(2.4 \%)$ & $72(2.1 \%)$ & $24(4.8 \%)$ & 0.0002 \\
\hline
\end{tabular}

Abbreviations: ICU, intensive care unit; MV, mitral valve; MVr, mitral valve repair; TV, tricuspid valve; TVr, tricuspid valve repair.

cohort. In the $\mathrm{MVr}+\mathrm{TVr}$ cohort, atrial fibrillation $(\mathrm{OR}=2.79$; 2.79; $\mathrm{CI}=1.01-9.89 ; p=0.0709)$, pulmonary hypertension $(\mathrm{OR}=2.69 ; \mathrm{CI}=1.12-6.73 ; p=0.0283)$, and arterial hypertension $(\mathrm{OR}=0.35 ; \mathrm{CI}=0.14-0.96 ; p=0.0301)$ were found to be most promising predictors.

\section{Discussion}

Motivation for the current investigation was a discussion on the augmentation of operative risk due to prolonged cardiopulmonary bypass time during MV repair with concomitant TV surgery due to the surgical access and surgical repair technique.

We investigated patients with minimally invasive MV repair with and without concomitant TV repair with an emphasis on surgery time, particularly cardiopulmonary bypass time, because TV repair was performed with either an arrested or a beating heart at the surgeon's discretion. In a previous study, we have shown that the postoperative outcomes were not different between patients who underwent isolated tricuspid surgery with a beating or an arrested heart. ${ }^{8}$ Furthermore, several previous studies have proposed that MV repair with concomitant TV repair is not associated with worse postoperative outcomes compared with isolated MV repair, which we were able to show as well. ${ }^{9-12}$

The preoperative parameters of patient cohorts in this investigation were different. We compared patients who underwent MV repair with or without concomitant TV repair. In patients with concomitant $\mathrm{TV}$ repair ( $\mathrm{MVr}+\mathrm{TVr}$ group), there were more patients with functional MV regurgitation, whereas in patients with isolated MV surgery (MVr group), there were more patients with structural MV regurgitation. Additionally, patients in the $\mathrm{MVr}+\mathrm{TVr}$ group were on an average 10 years older, had lower LVEF and higher atrial fibrillation, and had a higher rate of maze procedures than patients in the MVr group. However, on critically analyzing patients with functional versus structural MV surgery \pm maze procedure, we noted that the average difference in cardiopulmonary bypass time was usually $<40$ minutes in patients who underwent MV repair with and without concomitant TV repair in all subgroups. ICU stay and ventilation times were each around 5 hours prolonged in patients with additionally TV repair (after matching), which is significantly different but seems not to be clinically relevant.

We performed propensity score matching to enable direct comparison regarding early mortality. Furthermore, early mortality was not associated with prolonged cardiopulmonary bypass time.

Augmented operative risk during additional TV, specifically due to prolonged TV repair with a prosthetic ring, was not detected. Additionally, previous studies have shown that prosthetic annuloplasty rings are superior to suture techniques in TV repair. ${ }^{13,14}$

This investigation has some limitations due to the retrospective nature of the study and the heterogenous patient cohort. Additionally, detailed echocardiographic data are missing - for example regarding the extension of MV prolapse with or without annulus dilatation and informations about primary or secondary reasons for TV failure, or right ventricular pathology.

In conclusion, concomitant TV repair using prosthetic rings through a minimally invasive approach is safe and does not lead to elevated early mortality in our patient cohort. Therefore, prolonged cardiopulmonary bypass time should not be the sole reason to rule out MV repair with concomitant TV repair and to prefer the use of suture techniques, which saves only a few minutes compared with prosthetic ring implantation.

\section{Conflict of Interest}

None.

\section{References}

1 Falk V, Baumgartner H, Bax JJ, et al; ESC Scientific Document Group. 2017 ESC/EACTS Guidelines for the management of valvular heart disease. Eur J Cardiothorac Surg 2017;52(04):616-664

2 Tchantchaleishvili V, Rajab TK, Cohn LH. Posterior suture annuloplasty for functional tricuspid regurgitation. Ann Cardiothorac Surg 2017;6(03):262-265

3 Vahanian A, Alfieri O, Andreotti F, et al; Joint Task Force on the Management of Valvular Heart Disease of the European Society of Cardiology (ESC) and the European Association for Cardio-Thoracic Surgery (EACTS). Guidelines on the management of valvular heart disease (version 2012): the Joint Task Force on the Management of Valvular Heart Disease of the European Society of Cardiology (ESC) and the European Association for CardioThoracic Surgery (EACTS). G Ital Cardiol (Rome) 2013;14(03): 167-214

4 Kuntze T, Borger MA, Falk V, et al. Early and mid-term results of mitral valve repair using premeasured Gore-Tex loops ('loop technique'). Eur J Cardiothorac Surg 2008;33(04):566-572

5 Seeburger J, Borger MA, Falk V, et al. Minimal invasive mitral valve repair for mitral regurgitation: results of 1339 consecutive patients. Eur J Cardiothorac Surg 2008;34(04):760-765 
6 Seeburger J, Borger MA, Passage J, et al. Minimally invasive isolated tricuspid valve surgery. J Heart Valve Dis 2010;19(02): 189-192, discussion 193

7 Mohr FW, Falk V, Diegeler A, Walther T, van Son JA, Autschbach R. Minimally invasive port-access mitral valve surgery. J Thorac Cardiovasc Surg 1998;115(03):567-574, discussion 574-576

8 Pfannmüller B, Davierwala P, Misfeld M, Borger MA, Garbade J, Mohr FW. Postoperative outcome of isolated tricuspid valve operation using arrested-heart or beating-heart technique. Ann Thorac Surg 2012;94(04):1218-1222

9 Dreyfus GD, Corbi PJ, Chan KM, Bahrami T. Secondary tricuspid regurgitation or dilatation: which should be the criteria for surgical repair? Ann Thorac Surg 2005;79(01):127-132

10 Van de Veire NR, Braun J, Delgado V, et al. Tricuspid annuloplasty prevents right ventricular dilatation and progression of tricuspid regurgitation in patients with tricuspid annular dilatation under- going mitral valve repair. J Thorac Cardiovasc Surg 2011;141(06): 1431-1439

11 Chikwe J, Anyanwu AC. Surgical strategies for functional tricuspid regurgitation. Semin Thorac Cardiovasc Surg 2010;22(01): 90-96

12 Pfannmueller B, Verevkin A, Borger MA, et al. Role of tricuspid valve repair for moderate tricuspid regurgitation during minimally invasive mitral valve surgery. Thorac Cardiovasc Surg 2013; 61(05):386-391

13 McCarthy PM, Bhudia SK, Rajeswaran J, et al. Tricuspid valve repair: durability and risk factors for failure. J Thorac Cardiovasc Surg 2004;127(03):674-685

14 Navia JL, Nowicki ER, Blackstone EH, et al. Surgical management of secondary tricuspid valve regurgitation: annulus, commissure, or leaflet procedure? J Thorac Cardiovasc Surg 2010;139(06): 1473-1482.e5 